\title{
Antibody-targeted vaccination to lung dendritic cells generates tissue-resident memory CD8 Tcells that are highly protective against influenza virus infection
}

\author{
LM Wakim ${ }^{1}, \mathrm{~J} \mathrm{Smith}^{1}$, I Caminschi ${ }^{1,2}, \mathrm{MH}$ Lahoud $^{2,3}$ and JA Villadangos ${ }^{1,4}$
}

Influenza virus gains entry into the body by inhalation and initiates its replication cycle within the lung. The early stage of infection, while the virus is confined to the lung mucosa, provides the ideal window of opportunity for an effective immune response to control the infection. Tissue-resident memory (Trm) CD8 T cells, located in a variety of tissues including the lung, are ideally situated to act during this window and stall the infection. The factors involved in the differentiation of lung Trm cells remain poorly defined. We demonstrate that recognition of antigen presented locally by dendritic cells (DCs) and transforming growth factor- $\beta$ (TGF $\beta$ ) signaling are both required. We exploited this knowledge to develop an antibody-targeted vaccination approach to generate lung Trm cells. Delivering antigen exclusively to respiratory DCs results in the development of lung CD8 Trm cells that are highly protective against lethal influenza challenge. Our results describe an effective vaccination strategy that protects against influenza virus infection.

\section{INTRODUCTION}

Influenza virus infection represents a public health burden that results in significant morbidity and mortality. It is estimated to annually affect $5-10 \%$ of adults and $20-30 \%$ of children globally. The current inactivated influenza virus vaccines rely on the induction of neutralizing antibodies to viral surface glycoproteins. As these proteins mutate frequently, it is necessary to tailor new vaccines every year to the current strain of seasonal virus. ${ }^{1}$ In contrast, $\mathrm{CD} 8 \mathrm{~T}$-cell responses against invariable components of the virus provide protection against different strains. ${ }^{2-4}$ Vaccines that induce this type of protective immunity would provide major health and economic benefits.

For a $\mathrm{CD} 8{ }^{+} \mathrm{T}$ cell-based antiviral vaccine to protect against infection, the effector mechanism must be vigorous and capable of curtailing virus replication and spread early during the infection cycle. This is achieved in part by the ability of memory CD8 T cells to proliferate and gain cytotoxic function quickly upon detection of viral antigens. ${ }^{5}$ Until recently, it was assumed that memory $\mathrm{CD} 8 \mathrm{~T}$ cells were recruited to the infection site from the circulatory pool. Now it is well established that the skin and mucosal surfaces including the lung accumulate a specialized population of CD8 T cells, termed tissue-resident memory (Trm) cells, generated during previous antigenic challenges. ${ }^{6-9}$ These cells display a unique molecular signature that distinguishes them from the circulating memory $\mathrm{T}$ cell pool $^{10}$ and are long lived and self-sustaining. ${ }^{11}$. Trm cells are highly protective during localized infections, having the capacity to (i) directly kill pathogen-infected cells, ${ }^{12,13}$ (ii) release cytokines that render the surrounding/local environment nonpermissive for pathogen replication, and (iii) promote the recruitment of infection-fighting cells from the circulation. ${ }^{14}$

Influenza-specific CD8 Trm cells within the lung can protect against infection ${ }^{15,16}$ and have been shown to be highly abundant in the lungs of humans previously exposed to influenza virus, ${ }^{17}$ and hence a vaccination protocol that induces these cells may confer long-lasting immunity against this virus. ${ }^{1}$ The protocol

${ }^{1}$ Departments of Microbiology and Immunology, Doherty Institute of Infection and Immunity, The University of Melbourne, Melbourne, Victoria, Australia. ${ }^{2}$ Burnet Institute, Melbourne, Victoria, Australia. ${ }^{3}$ Department of Immunology, Monash University, Melbourne, Victoria, Australia and ${ }^{4}$ Department of Biochemistry and Molecular Biology, Bio21 Molecular Science and Biotechnology Institute, The University of Melbourne, Melbourne, Victoria, Australia. Correspondence: L Wakim or JA Villadangos (wakiml@unimelb.edu.au or j.villadangos@unimelb.edu.au) 
may comprise two steps: the first to activate naive antiinfluenza CD8 T cells (priming), and the second to promote the conversion of the primed cells to lung Trm cells. Multiple strategies for effective priming have been described, and the challenge now is to describe strategies that promote conversion of CD8 T cells to lung Trm cells. However, little is still known about the parameters that influence this conversion. Furthermore, the requirements for induction of Trm cells in different tissues vary. In the intestine, genital tract, or skin, entry of the effector cells into the tissue is sufficient to drive Trm cell formation. ${ }^{13,18}$ In contrast, formation of Trm cells in the nervous system or the lungs requires local antigen presentation. ${ }^{13,15,19}$ This raises the important question of which antigen-presenting cell (APC) is involved in Trm cell conversion in these tissues.

In this study we sought to characterize the factors that drive lung Trm cell formation following effective priming of an antiInfluenza virus CD8 T-cell response. We demonstrate that recognition of antigen presented locally by dendritic cells (DCs) and transforming growth factor- $\beta$ (TGF $\beta$ ) signaling are both required for the development of lung Trm cells. Utilizing an antibody-targeted vaccination strategy, we show that exclusively targeting antigen to respiratory DCs results in lung Trm cell development. The Trm cells generated by this vaccination regime were highly protective against lethal influenza infection.

\section{RESULTS \\ Local antigen recognition within the lung drives the differentiation of CD103 Trm cells}

Attracting effector CD8 T cells into the tissue by administering inflammatory agents ${ }^{13}$ or chemokines ${ }^{20,21}$ is sufficient to generate CD8 Trm cells in the skin, gut, or genital mucosa. Other tissues including the brain and dorsal root ganglia require local antigen recognition. ${ }^{13,19}$ To examine the antigen dependency of Trm cell differentiation in the lung, mice were infected intranasally with a parental strain of influenza virus (Flu) or a strain that had been engineered to express the model antigen OVA (Flu-OVA) 1 day before receiving in vitro activated ovalbumin (OVA)-specific OT-I CD8 ${ }^{+} \mathrm{T}$ cells (Figure 1a). Inoculating activated OT-I cells ensured that a large and equivalent number of effector cells, generated in identical conditions, were available for accurate measurements of conversion into Trm cells in the two groups of mice. Effector OT-I T cells are recruited to the lungs in the two conditions tested, but antigen recognition could only occur in mice that received Flu-OVA. After 20 days, these were the only mice that contained memory OT-I T cells expressing the Trm cell marker, CD103 (Figure 1b,c). This was also evident when OT-I cells were primed in vivo by injecting Flu-OVA into the footpad of mice 1 day before intranasal infection with either Flu-OVA or Flu (Supplementary Figure S1 online). Furthermore, mice seeded with in vitro-activated OT-I T cells before intranasal administration of inert inflammatory agents known to attract $\mathrm{T}$ cells to the lung (i.e., polyinosinic:polycytidylic acid or the chemokines CCL3/4) also failed to generate lung Trm cells (Supplementary Figure S2). This set of experiments indicates that, consistent with recent reports, ${ }^{15}$ conversion of effector cytotoxic T lymphocytes into Trm cells within the lung requires local antigen recognition.

\section{Bone marrow-derived cells can present antigen for Trm cell induction}

To gain insights into the identity of the APCs driving Trm cell formation, we generated bone marrow chimeras where only bone marrow-derived cells could present cognate antigen to OT-I T cells. Wild type (CD45.1) bone marrow was used to reconstitute lethally irradiated $\mathrm{H} 2-\mathrm{K}^{\mathrm{bml}}$ mice. The $\mathrm{H} 2-\mathrm{K}^{\mathrm{bm} 1}$ molecule cannot present the OVA-derived SIINFEKL epitope recognized by OT-I T cells, and hence in these chimeras only the bone marrow-derived compartment can present antigen to OT-I T cells. We adoptively transferred naive OT-I.CD45.1/2 $\mathrm{T}$ cells into these chimeric mice, infected the mice with FluOVA, and 20 days later determined the percentage of memory OT-I cells that converted into Trm cells within the lung. This percentage was equivalent to that observed in control chimeras or in normal C57BL/6 mice, in both of which all cells could present OVA (Figure 1d). Assessment of the anatomical location of the OT-I T cells in the lungs of these chimeric animals using intravascular staining ${ }^{9,22}$ confirmed that the Trm cells in all cohorts of mice were largely confined within the tissue, as they were less accessible to intravenous antibody staining than the circulating $\mathrm{CD} 8^{+} \mathrm{T}$ cells (Figure 1e,f). Therefore, the requirement for local antigen presentation to generate Trm cells can be fulfilled by bone marrow-derived cells, presumably professional APCs.

$\mathrm{CD}_{103}{ }^{+} \mathrm{DCs}$ promote $\mathrm{CD} 103$ upregulation upon CD8 T-cell activation in a TGF $\beta$-dependent manner

We next sought to identify the cross-presenting APCs that were driving Trm cell differentiation. The prime candidates were DCs and macrophages. To do this in the absence of the complexities associated with virus infection of a tissue, we transitioned to a model where we intranasally instilled whole OVA protein and an adjuvant into the airways of mice. This immunization regime results in the development of lung Trm cells (Figure 2a). Whole OVA protein and lipopolysaccharide (LPS) were intranasally instilled into the airways of animals and $18 \mathrm{~h}$ later we purified macrophages $\left(\mathrm{MHCII}^{+} \mathrm{F} 4 / 80^{+}\right.$), the two major DC populations within the murine lung, $\mathrm{CD} 11 \mathrm{~b}^{+}$and $\mathrm{CD}_{103}{ }^{+} \mathrm{DCs}$, and parenchymal cells as a negative control of cross-presentation capacity (Supplementary Figure S3). The cells were incubated with carboxyfluorescein succinimidyl ester (CFSE)-labeled OT-I cells and $60 \mathrm{~h}$ later we measured OT-I proliferation to determine which cell type cross-presented OVA. Both $\mathrm{CD}_{11 \mathrm{~b}^{+}}$and $\mathrm{CD} 103^{+}$DCs, and to a lesser extent macrophages, did (Figure 2b,c). Strikingly, the $\mathrm{CD}_{103}{ }^{+} \mathrm{DC}$ population was most efficient at inducing CD103 upregulation (Figure 2b,c). This was not simply a consequence of T-cell activation, as dividing OT-I cells "auto-activated" with SIINFEKL peptide alone failed to maintain expression of this integrin (Figure 2d). Rather, maintenance of CD103 expression was dependent on TGF $\beta$, a cytokine known to promote CD103 expression, as the introduction of TGF $\beta$ 
a
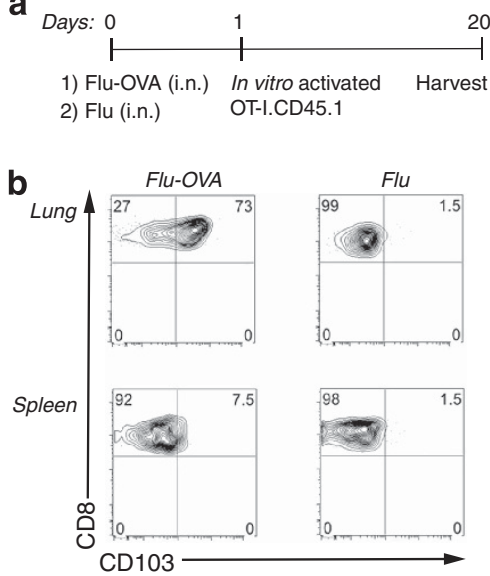

C

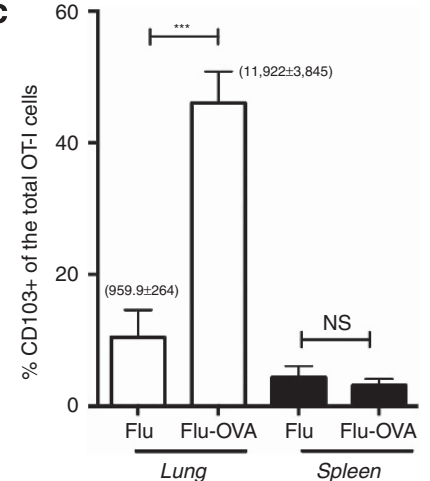

d
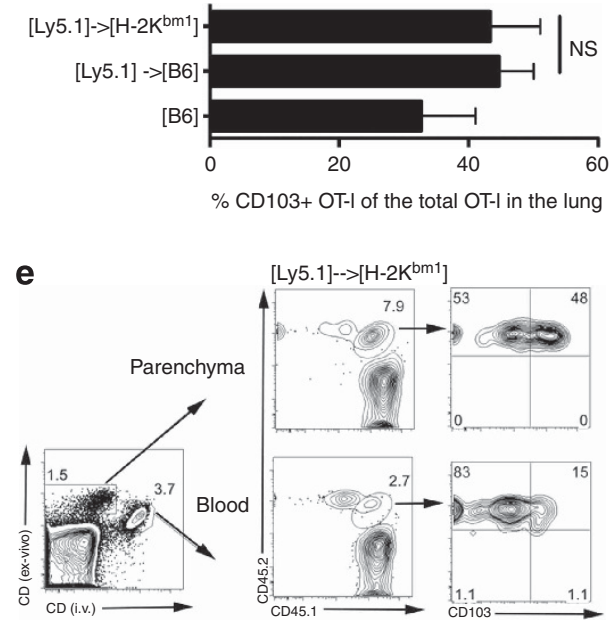

f

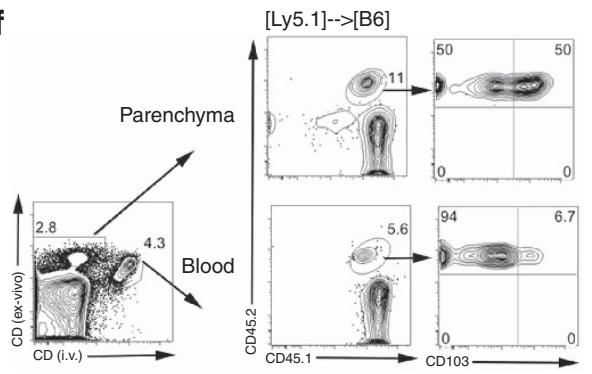

Figure 1 Local antigen recognition within the lung by a bone marrow-derived cell drives the differentiation of CD103 ${ }^{+}$tissue-resident memory (Trm) cells. (a) Experimental schematic. Mice were seeded with in vitro-activated OT-I.CD45.1 T cells 1 day after intranasal (i.n.) administration of either FluOVA (x31-OVA) or the parental strain Flu (x31). (b) Representative flow cytometry profiles gated on OT-I.CD45.1 ${ }^{+}$cells in the spleen and lung on day 20 after infection depicting the level of expression of CD103 for the indicated groups. (c) The proportion of CD103 ${ }^{+}$OT-I of the total OT-I T cells in the spleen and lung for the indicated groups 20 days after infection. Bars represent mean + s.e.m. $(n=6-7$ mice per group, pooled from three independent experiments. Numbers in the parenthesis represent the absolute number of cells \pm s.e.m.; Student's $t$-test, ${ }^{* * \star} P<0.0001$, nonsignificant (NS) $P>0.05$ ). (d) Naive OT-I.CD45.1/2 CD8 ${ }^{+} \mathrm{T}$ cells were transferred into [Ly5.1 $\rightarrow \mathrm{H} 2-\mathrm{K}^{\mathrm{bm} 1}$ ] or control [Ly5.1 $\left.\rightarrow \mathrm{B} 6\right]$ irradiation chimeras before intranasal infection with Flu-OVA (x31-OVA). The proportion of $\mathrm{CD}_{103^{+}}$OT-I of the total OT-I T-cell population within the lung was determined on day 20 after infection; bars represent mean + s.e.m. $(n=7-10$ mice per group, pooled from three independent experiments. Student's $t$-test, NS $P>0.05)$. (e, f) Naive OT-I.CD45.1/ $2 \mathrm{CD}^{+}{ }^{+}$cells were transferred into $(\mathbf{e})\left[\mathrm{Ly} 5.1 \rightarrow \mathrm{H} 2-\mathrm{K}^{\mathrm{bm} 1}\right]$ or $(\mathbf{f})[\mathrm{Ly} 5.1 \rightarrow \mathrm{B} 6]$ irradiation chimeras before intranasal infection with Flu-OVA $(\mathrm{x} 31-\mathrm{OVA})$. On day 20 after infection, mice were injected intravenously (i.v.) with anti-CD8 (CD8-iv) antibody. Mice were killed 5 min later and the levels of expression of CD103 on parenchyma-bound (CD8-iv ${ }^{\text {lo }}$ ) and blood-bound (CD8-iv ${ }^{\mathrm{hi}}$ ) OT-I.CD45.1/2 CD8 T cells within the lung were determined. Flow cytometry profiles representative of two independent experiments are shown.

neutralizing antibody into the assay blocked CD103 upregulation on the T cells (Figure 3a,b). We next assessed the source of the TGF $\beta$ in these cultures. TGF $\beta$ is produced in an inert form and requires processing to become biologically active. OT-I T cells activated with peptide alone did not synthesize significant amounts of biologically active TGF $\beta$, although large amounts of latent TGF $\beta$ could be detected in the culture supernatant, implying that these cell can synthesize but not efficiently activate this cytokine (Figure 3c). In contrast, the assessment of the amount of TGF $\beta$ - 1 secreted by LPS-stimulated respiratory DC subsets confirmed that the $\mathrm{CD}_{103}{ }^{+} \mathrm{DC}$ subset was proficient at producing both biologically active and latent TGF $\beta$ (Figure 3d,e). Assessment of the expression of the TGF $\beta$-activating integrin $\alpha(\mathrm{v}) \beta 8$ by flow cytometry and realtime-PCR revealed that although both $\mathrm{T}$ cells and DCs express integrin $\alpha \mathrm{V}(\mathrm{CD} 51)$ (Figure 3f), only DCs and particularly the $\mathrm{CD} 03^{+}$subset express the $\beta 8$ subunit (Figure 3g). Hence, upregulation of CD103 on the T cells may represent a coordinated response with both the $\mathrm{T}$ cells and DCs synthesizing latent TGF $\beta$ and the DCs processing it into its biologically active form. These results suggested that antigen cross-presentation by local DCs, in particular $\mathrm{CD}_{103}{ }^{+} \mathrm{DCs}$, induced Trm cells in the lung in a TGF $\beta$-dependent manner.

\section{$\mathrm{CD} 103^{+}$Trm cell formation in vivo is TGF $\beta$ dependent}

Next we assessed whether TGF $\beta$ signaling was required for lung Trm cell development in vivo. To do this, mice were seeded with naive OT-I T cells deficient in the expression of the TGF $\beta$ receptor. Mice were infected with Flu-OVA and 20 days later the level of expression of CD103 on the TGF $\beta R^{-1-}$ OT-I cells in the lung was determined. These cells failed to upregulate the integrin CD103, indicating a requirement for TGF $\beta$ signaling 
a
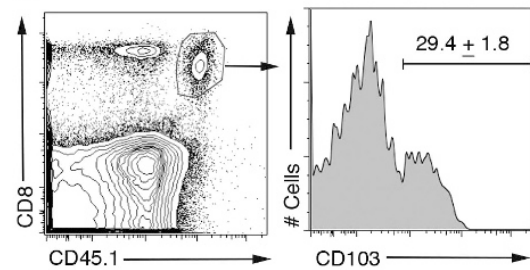

b
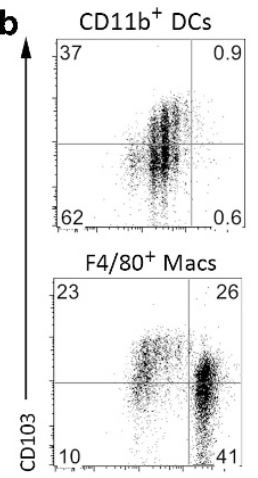

CFSE c
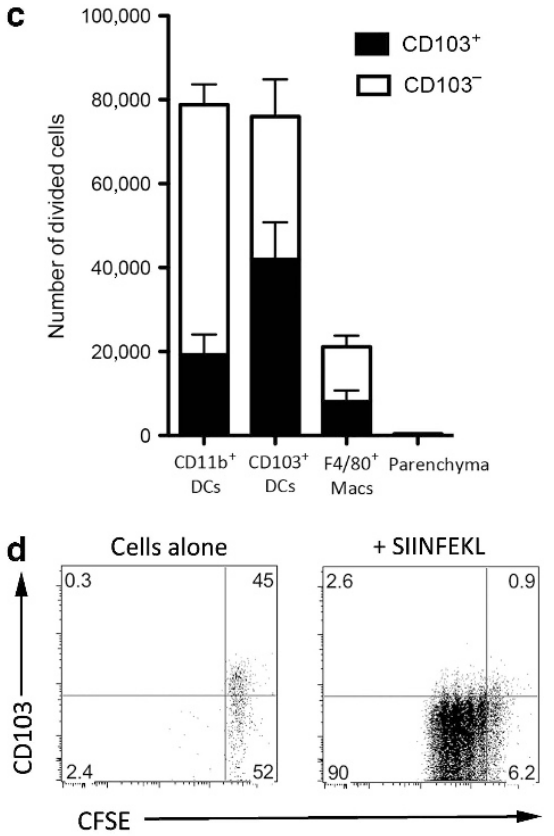

Figure $2 \mathrm{CD}_{103}{ }^{+}$respiratory dendritic cells (DCs) preferentially drive CD103 expression upon CD8 T-cell activation. (a) Mice were seeded with in vitroactivated OT-I.CD45.1 T cells 1 day before intranasal delivery of ovalbumin (OVA) protein + lipopolysaccharide (LPS). Representative flow cytometry profiles depicting the level of expression of CD103 on OT-I cells in the lung 20 days after OVA administration is shown. Number within the histograph represents the mean + s.e.m. ( $n=6$, data are representative of three independent experiments). (b) CD $11 b^{+}$and $C D 103^{+} \mathrm{DCs}$, F4/80 ${ }^{+}$macrophages, and parenchyma cells were sorted from the lungs of mice $18 \mathrm{~h}$ following intranasal instillation of LPS + OVA and were cultured with carboxyfluorescein succinimidyl ester (CFSE)-labeled OT-I cells. The number of divided cells in the culture was enumerated $60 \mathrm{~h}$ later. Representative flow cytometry profiles depicting the CFSE proliferation profiles and the level of expression of CD103 on the divided cells. (c) The absolute number of CD103 ${ }^{+}$and $\mathrm{CD} 103^{-}$ divided cells in the culture. Bars represent mean + s.e.m. ( $n=5$ mice pooled per experiment, data pooled from five independent experiments). (d) CFSElabeled naive OT-I cells were cultured alone or in the presence of $10^{-6} \mathrm{M}$ SIINFEKL for $60 \mathrm{~h}$. Representative flow cytometry profiles depict CFSE division and CD103 expression on the divided cells. Data representative of three independent experiments.

(Figure 4a,b). Strikingly, intravascular staining showed that whereas $>80 \%$ of wild type OT-I memory T cells were located within the parenchymal lung tissue (stained negative for the intravenously injected antibody), only $27 \%$ of the TGF $\beta$-deficient OT-I cells were in this location (Figure 4c). This indicated that TGF $\beta$ was not only required to induce CD103 expression but also to promote accumulation within tissues, the hallmark of Trm cells.

\section{Antibody-targeted vaccination induces lung-resident memory $\mathrm{T}$ cells}

Our results above established the importance of local antigen presentation and TGF $\beta$ signaling for cytotoxic T lymphocyte conversion into lung Trm cells, and the differential role of different APCs in TGF $\beta$ production and activation. We sought to exploit this knowledge to devise a vaccination protocol to safely induce protective immunity against lethal influenza virus infection by depositing influenza-specific Trm cells within the respiratory tract. Indeed, excellent protection was generated against genital herpes simplex virus 2 infection by applying chemokines to the genital mucosa to "pull" effector CD8 T cells to promote their differentiation into Trm cells within the genital tract. ${ }^{21}$ This strategy would not be effective at depositing Trm cells within organs like the lung where local antigen presentation is a requirement for Trm cell generation.
Therefore, we decided to investigate whether it would be possible to generate anti-influenza virus-specific Trm cells by incorporating local antigen presentation into the pull step.

To promote local antigen presentation in the lung, we employed "antibody-targeted vaccination" (ATV). ATV involves the inoculation of antigen coupled to monoclonal antibodies (mAbs) against DC receptors. It has been previously shown to promote effective cross-presentation by DCs in vivo. ${ }^{23-25}$ When administered together with an adjuvant such as LPS, this approach leads to effective cross-priming of naive CD8 T cells, but it is not known whether ATV can be delivered by the intranasal route and whether it can boost conversion of cytotoxic T lymphocytes into Trm cells.

Analysis of the DCs contained in the lungs of mice exposed to LPS showed that two receptors that have previously been used as targets for ATV, DEC205 and Clec12A, were differentially expressed in $\mathrm{CD}_{103}{ }^{+}$and $\mathrm{CD}_{11 \mathrm{~b}}{ }^{+}$DCs. Both subsets expressed Clec12A equivalently but DEC205 was expressed only by $\mathrm{CD}_{103}{ }^{+}$DCs (Figure 5a). We assessed whether the intranasal delivery of anti-Clec12A and anti-DEC205 would target these lung DC subsets. Mice were administered intranasally anti-Clec12A, anti-DEC205, or an isotype control antibody conjugated to a fluorescent dye. After $1 \mathrm{~h}$, we extracted the DCs from the lungs of these mice and assessed which subset was labeled with the fluorescent antibody. Consistent with our 
a

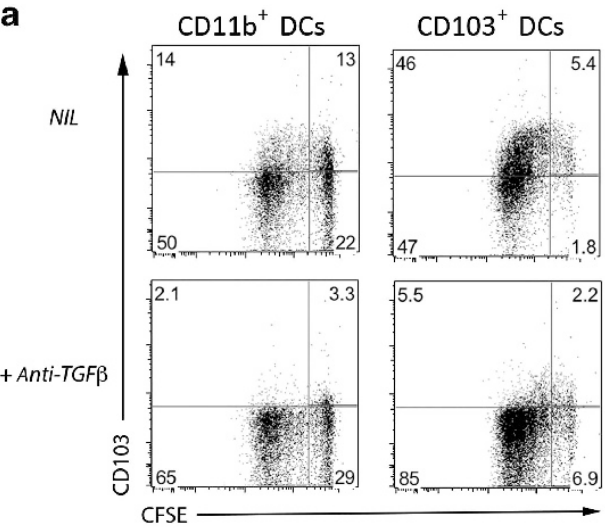

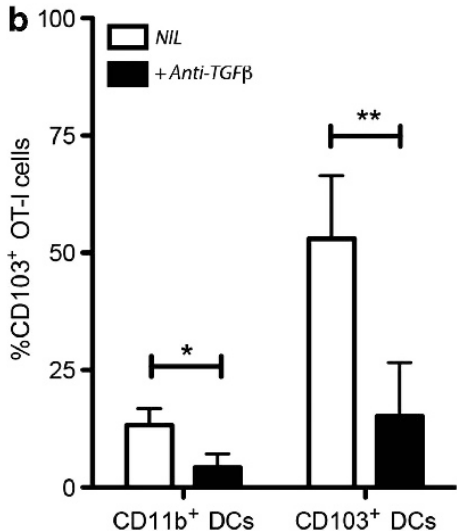

e

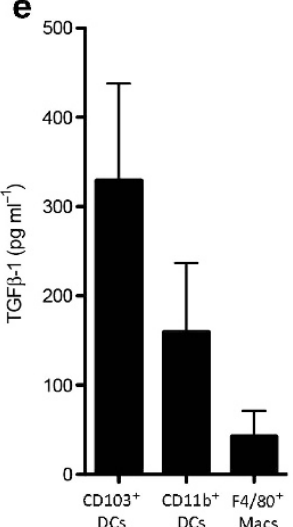

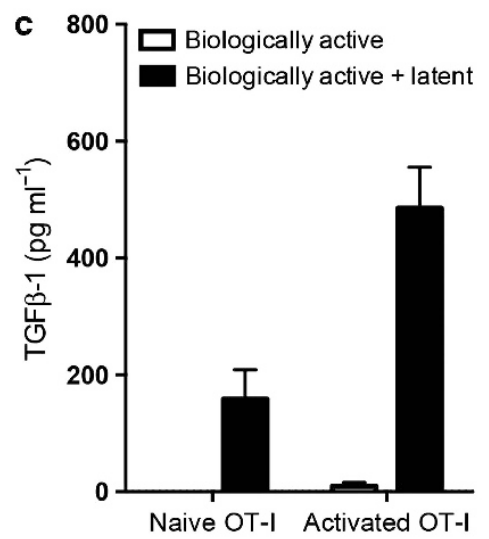

f

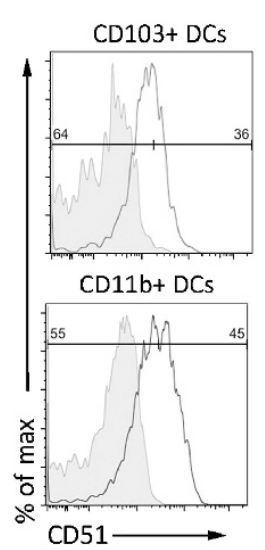

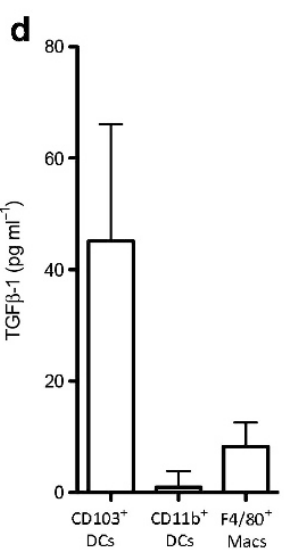
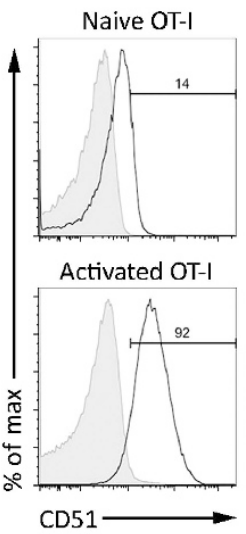

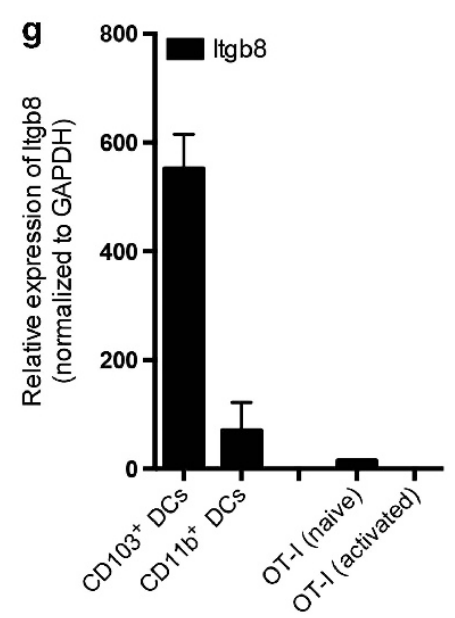

Figure 3 Respiratory dendritic cells (DCs) preferentially drive CD103 expression upon CD8 T-cell activation in a transforming growth factor- $\beta$ (TGF $\beta$ )dependent manner. (a) $\mathrm{CD}_{11} \mathrm{~b}^{+}$and $\mathrm{CD} 103^{+} \mathrm{DCs}$ were sorted from the lungs of mice $18 \mathrm{~h}$ following intranasal instillation of lipopolysaccharide (LPS) + ovalbumin (OVA) and were cultured with carboxyfluorescein succinimidyl ester (CFSE)-labeled OT-I cells in the presence or absence of antiTGF $\beta$ neutralizing antibody. Representative flow cytometry profiles depicting the CFSE proliferation profiles and the level of expression of CD103 on the divided cells $60 \mathrm{~h}$ later are shown. (b) Bars represent the mean + s.e.m. $(n=5$ mice pooled per experiment, data pooled from three independent experiments. Student's $t$-test, ${ }^{\star} P<0.03,{ }^{\star \star} P<0.006$, nonsignificant (NS) $P>0.05$ ). (c) Naive OT-I cells were cultured alone or in the presence of $10^{-6} \mathrm{M}$ SIINFEKL for $60 \mathrm{~h}$. The amount of biologically active and total TGF $\beta$ (latent and biologically active) in the supernatants was measured by enzyme-linked immunosorbant assay (ELISA). Data are pooled from four independent experiments. Bars represent mean + s.e.m. (d, e) CD11b ${ }^{+}$and CD103 ${ }^{+}$DCs and $\mathrm{F} 4 / 80^{+}$macrophages were sorted from the lungs of mice and cultured overnight with LPS. The amount of (d) biologically active and (e) total TGF $\beta$ (latent and biologically active) in the supernatants was measured by ELISA. Data are pooled from three independent experiments. Bars represent mean + s.e.m. (f) Representative flow cytometry depicting the level of expression of CD51 on $\mathrm{CD}_{103^{+}}$and CD11b ${ }^{+}$DCs isolated from the lung, naive OT-I cells, and OT-I cells on day 3 after activation. Gray histograph represents isotype control staining. (g) The level of expression of itgb8 mRNA on the subsets described above. Data are pooled from three independent experiments and expression levels are normalized to the housekeeping gene glyceraldehyde 3-phosphate dehydrogenase (GAPDH). 

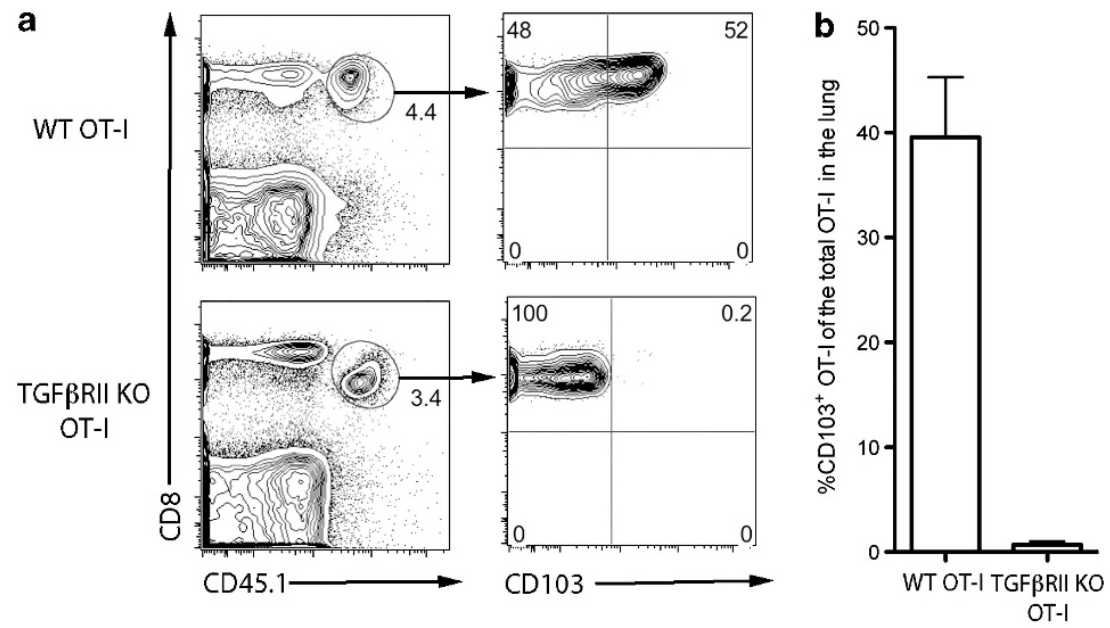

C
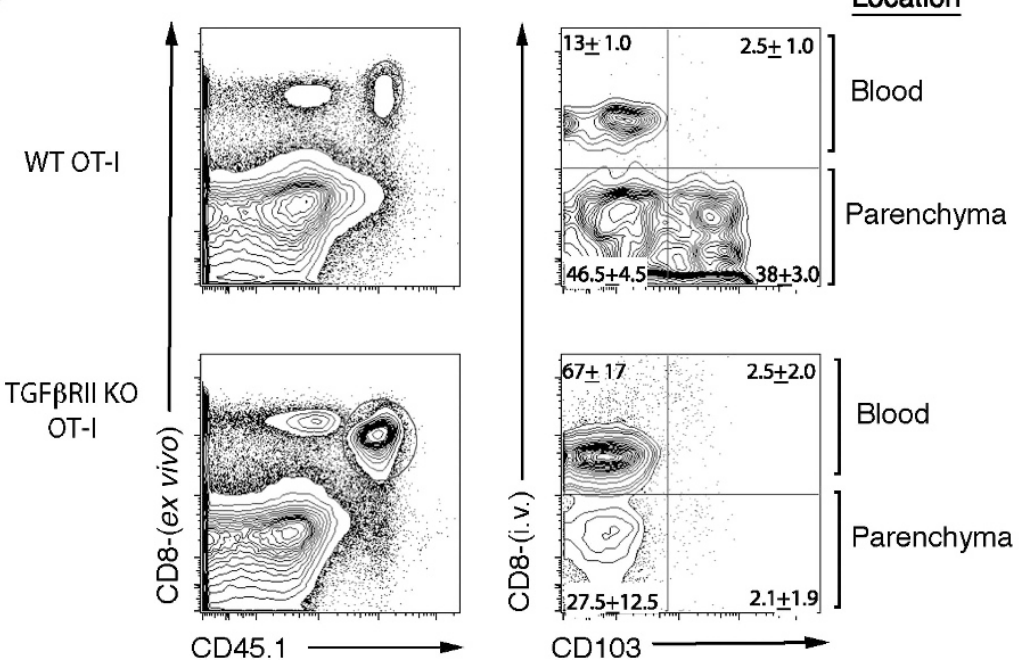

Figure 4 Generation of lung tissue-resident memory (Trm) cells is dependent on transforming growth factor- $\beta$ (TGF $\beta$ ) signaling. Mice were seeded with naive OT-I.TGF $\beta R^{-1-}$.CD45.1 or wild-type OT-I.CD45.1 cells before intranasal infection with Flu-OVA (x31-OVA). On day 20 after infection, the percentage of OT-I cells expressing CD103 was measured. (a) Representative flow cytometry profiles displaying the level of expression of CD103 on the OT-I cells. (b) Data are pooled from three independent experiments, $n=16$. Bars represent mean + s.e.m. (c) Mice were seeded with naive OTI.TGF $\beta R^{-I-}$.CD45.1 or wild-type OT-I cells before intranasal infection with Flu-OVA (x31-OVA). On day 20 after infection, mice were injected intravenously (i.v.) with anti-CD8 (CD8-iv) antibody. Mice were killed 5 min later and the levels of expression of CD103 on parenchyma-bound (CD8-iv ${ }^{\text {lo }}$ ) and blood-bound (CD8-iv ${ }^{\text {hi }}$ ) OT-I CD8 T cells within the lung were determined. Flow cytometry profiles are representative of two independent experiments. Numbers shown in the quadrants represent mean \pm s.e.m.; $n=4-6$.

in vitro staining, intranasal delivery of anti-Clec12A labeled both DC subsets within the lung, whereas the intranasal delivery of anti-DEC205 labeled only the $\mathrm{CD} 103^{+}$DC subset (Figure 5b,c). This demonstrates, consistent with prior reports, ${ }^{26}$ that ATV delivered via the intranasal route is a feasible strategy to target antigen to lung DCs.

We next sought to determine whether we could target antigen to the respiratory DCs to generate Trm cells using antiDec205 and anti-Clec12a mAbs linked to OVA. The purpose of this experiment was to test the relative efficacy of each $\mathrm{mAb}$ as a strategy to promote local antigen presentation within the lung to anti-OVA-specific $\mathrm{T}$ cells to promote the development of Trm cells, and hence it was important to compare mice with equally large numbers of primed cells in order to carry out accurate measurements of local conditioning. To do this, we inoculated mice intravenously with in vitro-activated OT-I cells before intranasal delivery of 1 or $5 \mu \mathrm{g}$ of either mAb-OVA conjugate together with LPS. Control mice received isotype control antibody conjugated to OVA plus LPS, or LPS alone. After 20 days, we enumerated the OT-I Trm cells within the lungs of each group of mice (Figure 6a). The mice that received ATV contained 2.5-5-fold more OT-I cells in the lung than those treated with LPS alone (Figure 6b). Moreover, the proportion of OT-I contained in the lung that expressed the Trm cell marker CD103 was higher in mice that received anti-Clec12A-OVA or anti-DEC205-OVA (Figure 6c). Importantly, all cohorts of mice had similar proportions of memory OT-I cells within the spleen (Figure 6d), indicating that the local delivery of OVA-conjugated antibodies to the airways did not result in boosting of the systemic OT-I T cell pool. 
a

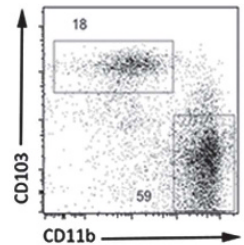

$\mathrm{CD}_{103^{+}} \mathrm{DCs}$

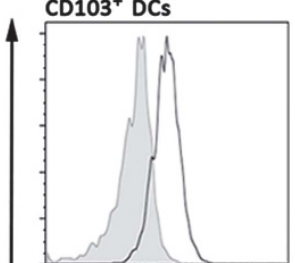

CD11b ${ }^{+}$DCs

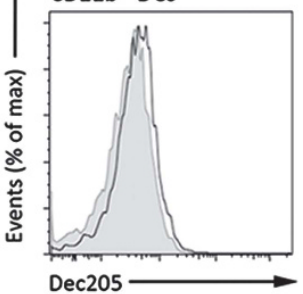

CD103+ DCs

( $\left.\mathrm{F} 4 / 80^{-} \mathrm{MHCII}{ }^{+} \mathrm{CD} 11 \mathrm{C}^{+} \mathrm{CD} 11 \mathrm{~b}^{-} \mathrm{CD}^{-103^{+}}\right)$

CD11b+ DCs

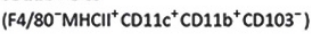
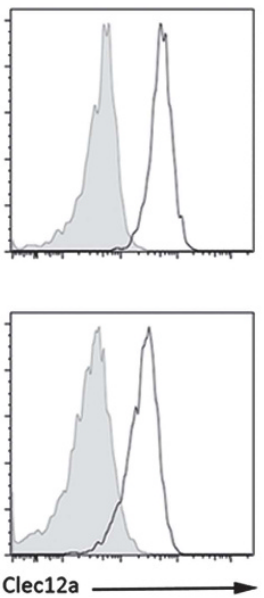

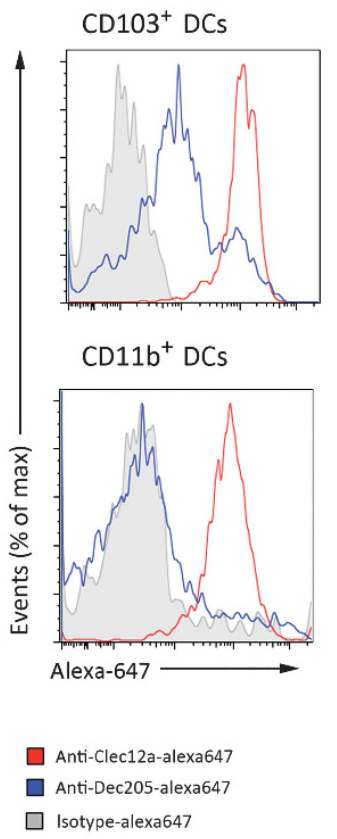

\section{b}
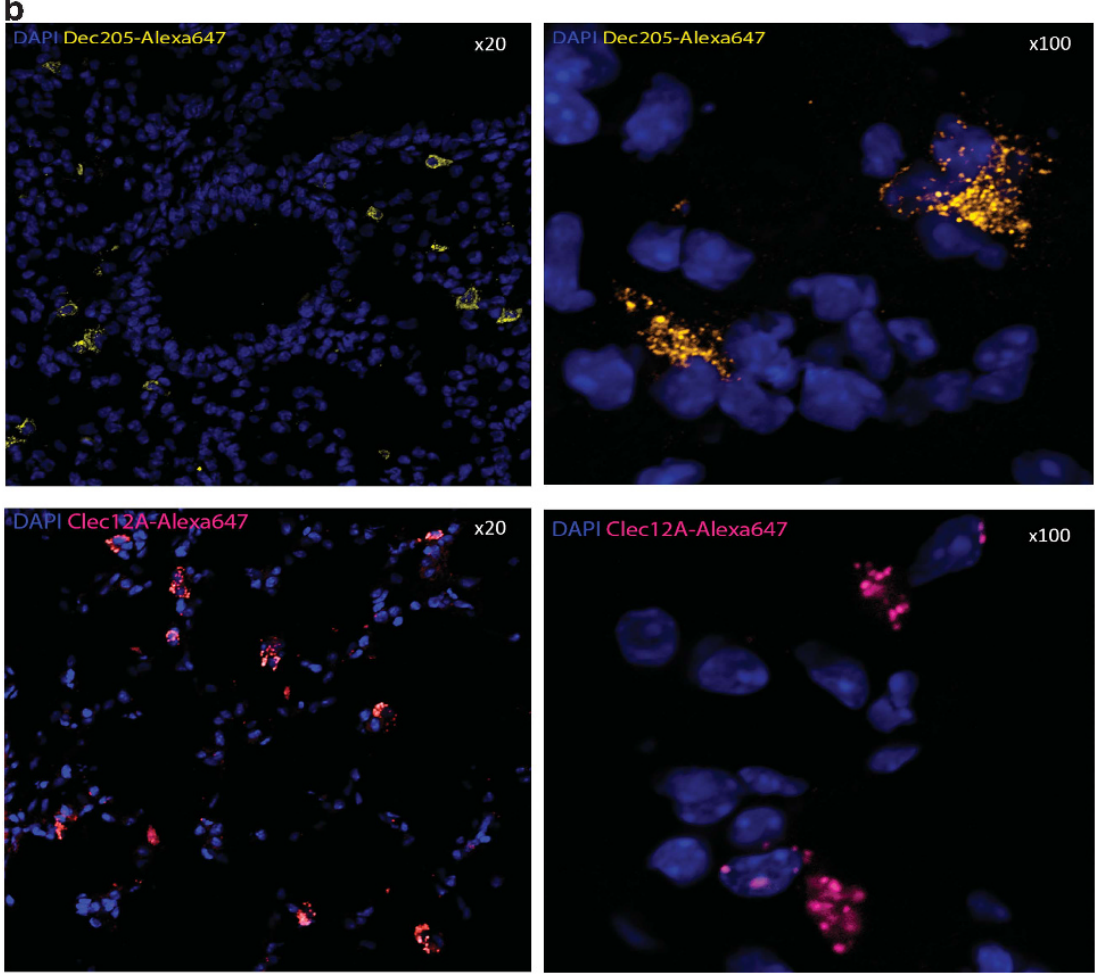

Figure 5 Intranasal delivery of antibodies specific for dendritic cell (DC) surface molecules DEC205 and Clec12A specifically targets respiratory DCs within the lung mucosa. (a) Representative flow cytometry profiles depicting the level of expression of Clec12A and DEC205 on CD11b ${ }^{+}$(F4/ $\left.80^{-} \mathrm{MHClI}{ }^{+} \mathrm{CD} 11 \mathrm{c}^{+} \mathrm{CD}_{11 \mathrm{~b}^{+}}\right)$and $\mathrm{CD}_{103}{ }^{+}\left(\mathrm{F} 4 / 80^{-} \mathrm{MHCII}{ }^{+} \mathrm{CD} 11 \mathrm{C}^{+} \mathrm{CD} 103^{+}\right) \mathrm{DCs}$ isolated from the lung $24 \mathrm{~h}$ after intranasal lipopolysaccharide $(\mathrm{LPS})$ administration. Shaded histograms represent isotype control. Data are representative of three experiments. (b, c) Mice were administered intranasally either anti-Clec12A or anti-DEC205 or isotype conjugated to Alexa-647. Lungs were recovered and the level of Alexa-647 fluorescence on the CD103 and $C D 11 b^{+}$DC subsets were determined by (b) confocal microscopy and (c) flow cytometry. Data are representative of three independent experiments.

The generation of lung Trm cells can be considered a twophase process in terms of antigen presentation. The first phase of antigen presentation occurs within the lymph node and acts to prime naive $\mathrm{T}$ cells, whereas the second phase, which occurs locally within the lung, programs Trm cell differentiation. A vaccination regime would need to support both phases of 
a

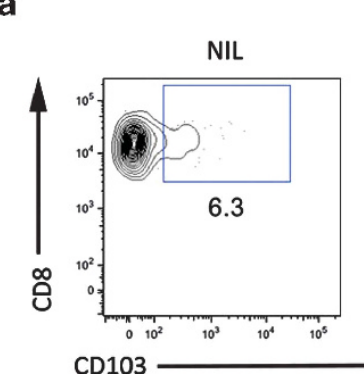

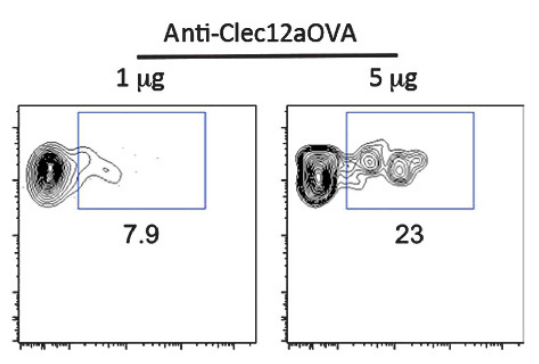

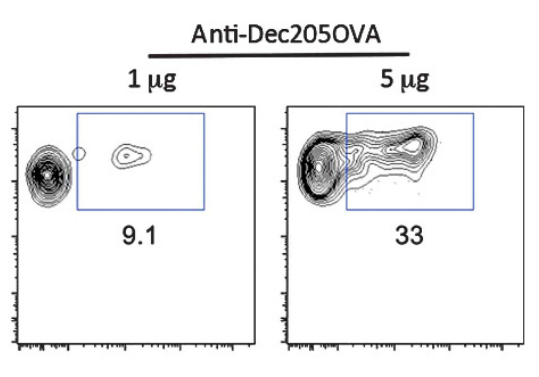

b

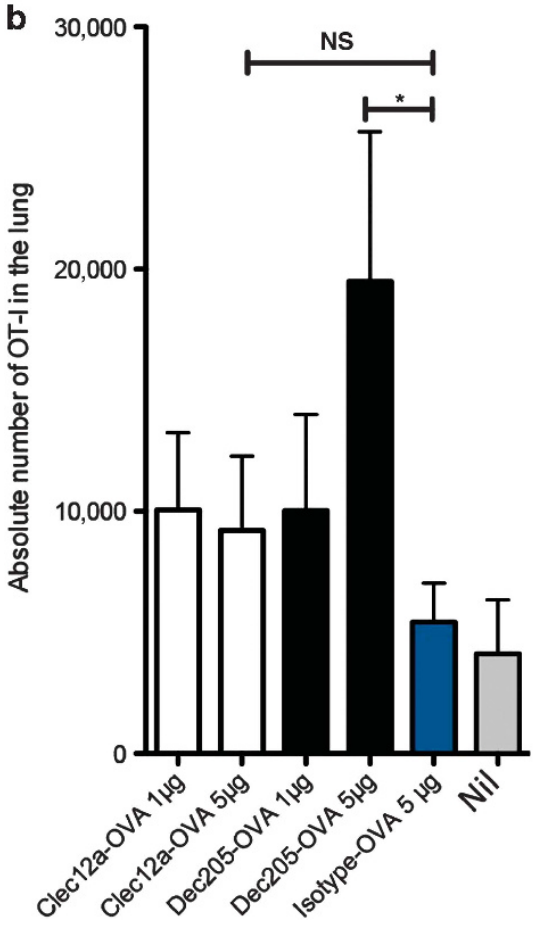

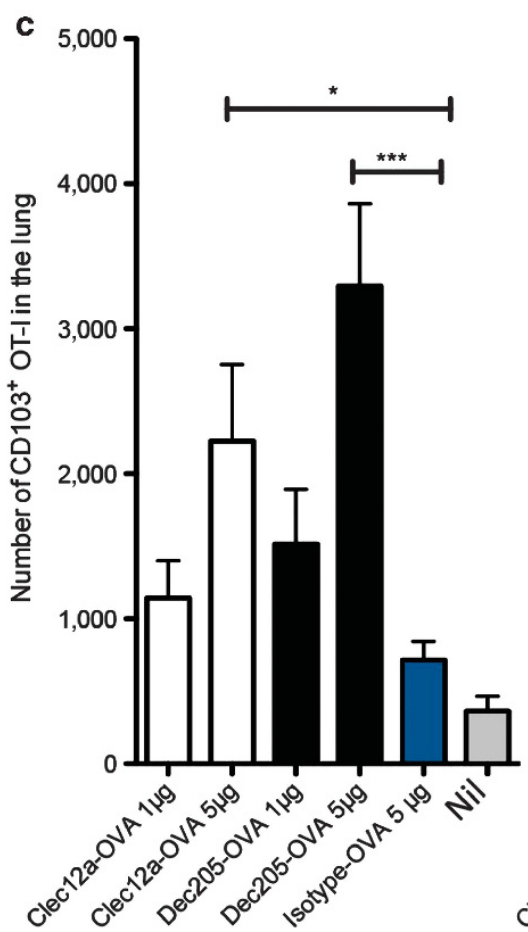

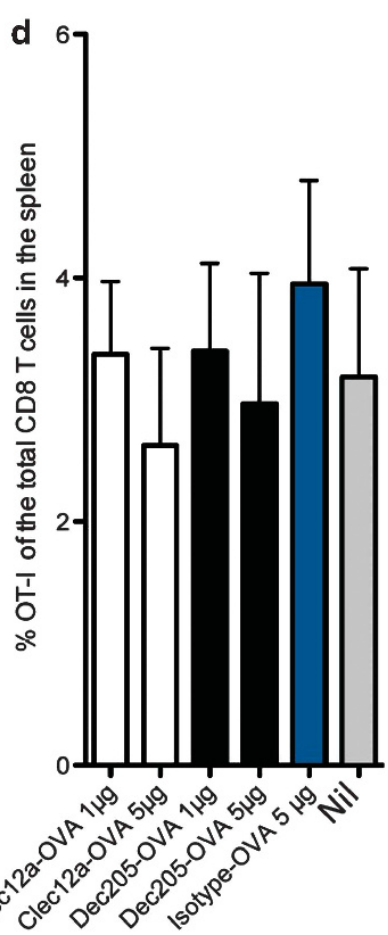

Figure 6 Targeting protein antigen to lung dendritic cells (DCs) using antibody-targeted vaccination (ATV) results in the development of CD103 ${ }^{+}$ tissue-resident memory (Trm) cells. Mice were seeded with in vitro-activated OT-I.CD45.1 T cells before intranasal administration of lipopolysaccharide (LPS) and either anti-Clec12a-OVA, anti-Dec205-OVA, isotype-OVA, or phosphate-buffered saline (PBS) (Nil). (a) Representative flow cytometry profiles showing the level of expression of CD103 on OT-I cells in the lung 20 days after antibody administration. (b-d) The absolute number of (b) OT-I cells or (c) OT-I.CD103 ${ }^{+}$cells in the lung in the indicated groups of mice on day 20 following antibody administration $(n=7-16$ mice per group, data pooled from five independent experiments. One-way analysis of variance (ANOVA), with Bonferroni's multiple comparisons, ${ }^{\star} P<0.05$, ${ }^{* \star *} P<0.001$, nonsignificant (NS) $P>0.05$ ). (d) Percentage OT-I of the total CD8 ${ }^{+}$T-cell population in the spleen in the indicated groups; bars represent mean + s.e. $m$.

antigen presentation. We assessed the ability of ATV therapy to both prime $\mathrm{CD} 8 \mathrm{~T}$-cell responses and promote Trm cell formation. First, we assessed whether ATV administered by the intranasal route could promote $\mathrm{T}$-cell priming in the draining lymph node. To do this, mice were seeded with naive CFSElabeled OT-I before intranasal delivery of $5 \mu \mathrm{g}$ of anti-Clec12aOVA or anti-Dec205-OVA. Assessment of OT-I division within the lung draining lymph node, measured by the dilution of CFSE 4 days following immunization, revealed that both targeting antibodies could effectively promote T-cell division (Supplementary Figure S4a). We next assessed whether immunization with these mAb-OVA could both prime CD8 $\mathrm{T}$-cell responses and promote Trm cell formation. To do this, mice were seeded with low numbers of naive OT-I cells 1 day before intranasal delivery of OVA-conjugated antibodies with adjuvant. The number of OT-I cells in the lung expressing CD103 was measured 20 days later. Immunization with antiDec205-OVA or anti-Clec12a-OVA resulted in a 4-6-fold increase in the number of $\mathrm{CD}_{103}{ }^{+}$OT-I cells in the lung compared with animal receiving the isotype control antibody (Supplementary Figure S4b).

\section{ATV induces protective immunity against influenza virus infection}

We next tested the protective capacity of Trm cells induced by ATV in the lung. To do this, mice were seeded with in vitroactivated OT-I cells and immunized with LPS alone or with $5 \mu \mathrm{g}$ of either DEC205-OVA or Clec12a-OVA to induce Trm cells within the lung. We utilized this regime as all cohorts of mice had equal-sized populations of circulating memory OT-I 

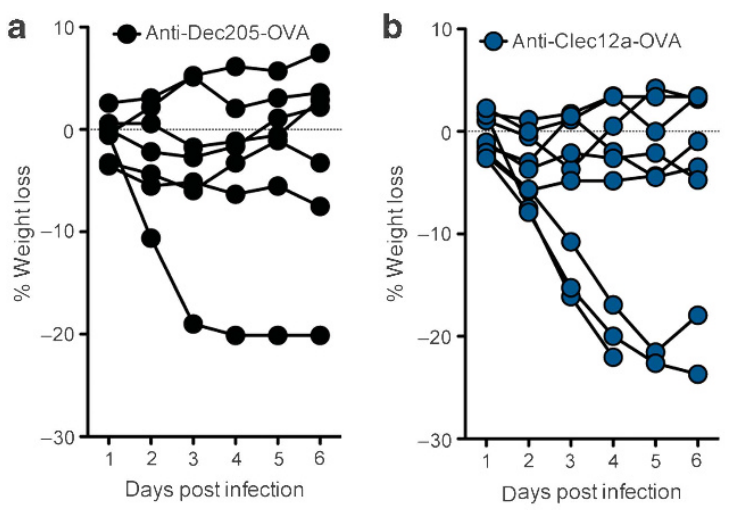

g
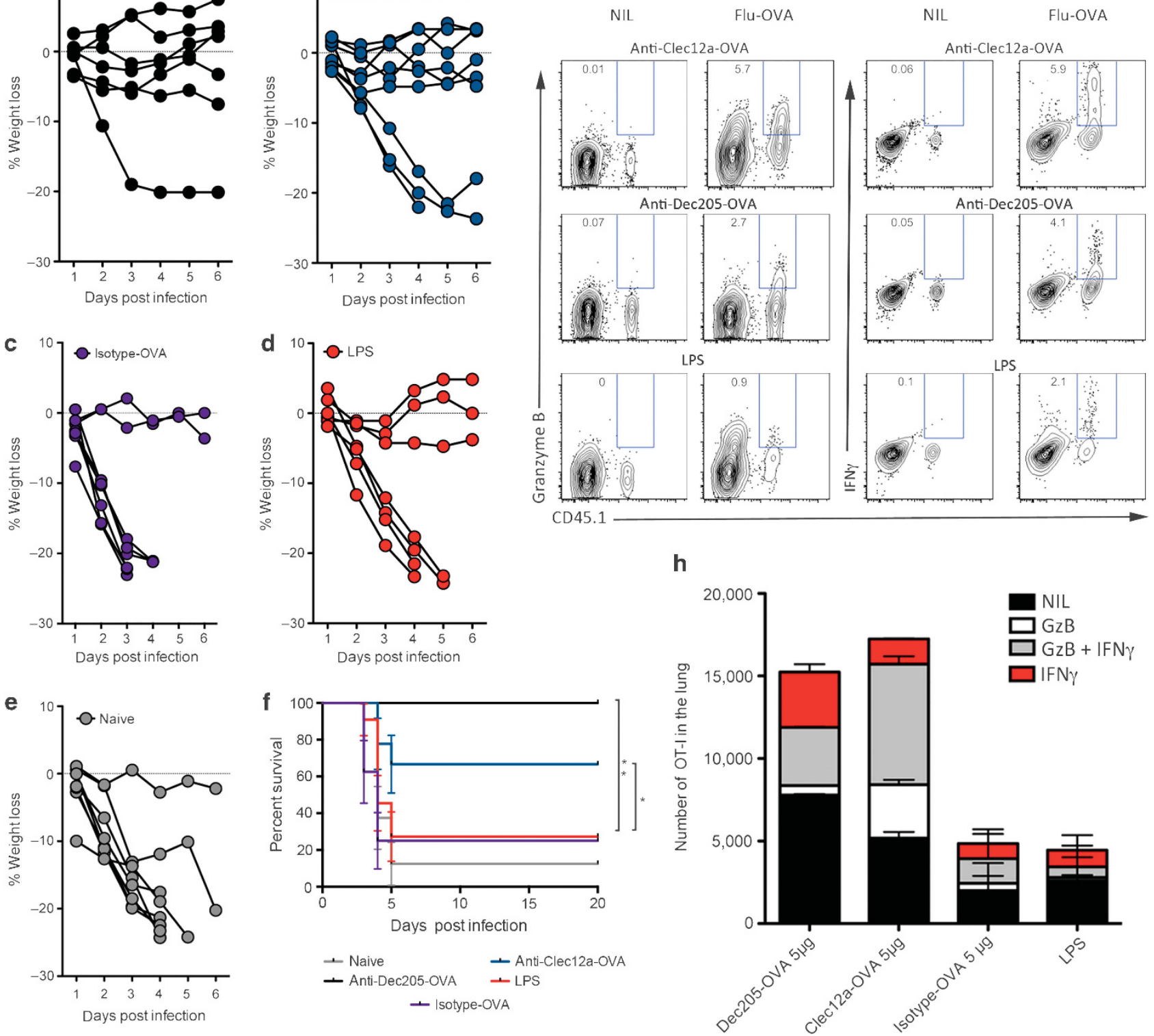

Figure 7 Lung tissue-resident memory (Trm) cells generated using antibody-targeted vaccination (ATV) results in the development of CD103 ${ }^{+}$Trm cells that are protective against lethal influenza challenge. Mice were seeded with in vitro-activated OT-I.CD45.1 T cells before intranasal administration of lipopolysaccharide (LPS) and $5 \mu \mathrm{g}$ of (a) anti-Dec205-OVA, (b) anti-Clec12a-OVA, (c) isotype-OVA, (d) or phosphate-buffered saline (PBS) alone. After 20 days, these mice and (e) a naive cohort were challenged with Pr8-OVA. Graphs depict weight loss curves for individual animals. (f) The percentage survival (mice losing $<20 \%$ body weight) was monitored. Graphs depict Kaplan-Meier survival curves. Data are pooled from four independent experiments with $n=7-9$ mice per group. Log-rank (Mantel-Cox) test, ${ }^{\star} P<0.0427,{ }^{\star *} P<0.004$. (g) Mice were seeded with in vitro-activated OT-I.CD45.1 T cells before intranasal administration of LPS and $5 \mu \mathrm{g}$ of the indicated targeting antibody. After 20 days, these mice were challenged with Flu-OVA (Pr8-OVA) or given PBS (NIL) and the proportion of Granzyme B (GzB)- and interferon- $\gamma$ (IFN $\gamma$ )-producing OT-I.CD45.1 in the lung was measured directly ex vivo on day 2 after challenge. (g) Representative flow cytometry profiles gated on total CD8 T cells in the lung. (h) Graph depicts the absolute number of OT-I cells in the lung producing varying combinations of cytokines $(n=5-6$ mice per group, data pooled from two independent experiments).

T cells, and therefore this would allow assessment of how specifically increasing lung Trm cells would impact control of influenza virus. The mice were challenged 20 days later with highly virulent Flu-OVA influenza virus (PR8-OVA). All mice immunized with anti-DEC205-OVA (Figure 7a,f), and 66\% of those immunized with anti-Clec12a-OVA (Figure $7 \mathbf{b}$ and $\mathbf{f}$ ) survived the challenge compared with $33-40 \%$ of the mice that received LPS or isotype-OVA alone (Figure $7 \mathbf{c}, \mathbf{d}, \mathbf{f})$ and just $12.5 \%$ of those that received no OT-I cells (Figure 7e,f). Survival curves correlated with a reduction in viral titers in the lung as measured on day 4 after infection (Supplementary Figure S5). As all three non-naive groups of mice contained equivalent numbers of circulating memory OT-I cells (Figure 6d), these results demonstrate that the number of 
Trm cells in the lung mucosa is more important in protection against a viral challenge than the number of circulating memory cells. To gain insights into the features that make the Trm cells induced by ATV highly protective, we analyzed the production of two major effector molecules, Granzyme B and interferon- $\gamma$, by OT-I cells found in the lungs of the mice 2 days after infection. Mice vaccinated with anti-Dec205OVA and anti-Clec12a-OVA contained 3-5 times more OT-I cells producing interferon- $\gamma$, Granzyme B, or both than mice vaccinated with isotype control conjugate or that received LPS alone (Figure $\mathbf{7 g}-\mathbf{h}$ ). ATV is thus an effective regimen for the generation of highly protective Trm cells within the lung.

\section{DISCUSSION}

Influenza virus is a major burden on public health worldwide. To maintain protective immunity against influenza virus, the current vaccines need to be annually tailored to match the current strain of seasonal virus. For no other pathogen is there this requirement for yearly immunization to maintain protective immunity. Furthermore, vaccinated individuals are not protected in the event of a pandemic outbreak. A universal vaccine that provides sustained, prophylactic protection against multiple strains of seasonal and pandemic influenza would provide major health and economic benefits.

The induction of long-lived memory CD8 ${ }^{+} \mathrm{T}$-cell immunity is an effective means of inducing cross-protection against different influenza strains. Trm cells are ideally situated to mediate this protection and hence strategies that generate Trm cells within the lung hold significant prophylactic potential. Here we showed that the generation of lung Trm cells is dependent on local antigen presentation and TGF $\beta$ signaling. This is consistent with earlier reports by Lee et al. ${ }^{27}$ where it was shown that $\mathrm{T}$ cell receptor and TGF $\beta$ signaling are required for CD69 and CD103 upregulation on effector T cells infiltrating influenza virus-infected lungs. Although the requirement for TGF $\beta$ signaling appears to be a universal requirement for Trm cell development in all tissues, including the gut, skin, and genital mucosa, ${ }^{18,28,29}$ the requirement for local antigen presentation is more variable. ${ }^{30} \mathrm{~A}$ possible explanation for this variability is that high levels of TGF $\beta$ may bypass the requirement for antigen presentation. This may be the case in the intestine, where this cytokine is highly expressed. The concentration of TGF $\beta$ in other tissues such as the lung is lower, and for effector CD8 $\mathrm{T}$ cells to detect TGF $\beta$ and undergo differentiation into Trm cells it may be necessary for the participation of an accessory cell to "concentrate" the cytokine. DCs may play this role. Utilizing a humanized mouse model, it was demonstrated that lung-resident $\mathrm{CD}_{1} \mathrm{c}^{+} \mathrm{DCs}$ drove CD103 expression on effector $\mathrm{CD} 8{ }^{+} \mathrm{T}$ cells by displaying membrane-bound TGF- $\beta 1 .^{31}$ Similarly, the murine CD103 ${ }^{+}$ DC population appears to be particularly adept at performing this function, plausibly because in addition to secreting TGF $\beta$, it can cross-present the antigens recognized by effector CD8 $\mathrm{T}$ cells that may promote the establishment of stable intercellular interactions and thus facilitate interactions with TGF $\beta$.
Inoculation of antigen coupled to mAbs against endocytic receptors on DCs (ATV) has been successfully utilized to promote CD8 T-cell priming. ${ }^{25,32}$ Here we use this therapy effectively to target antigen to mucosal DC subsets, not to initiate T-cell priming, but to promote local antigen presentation as a method to condition effector $\mathrm{T}$ cells locally and drive their differentiation into lung Trm cells. This work highlights the use of ATV as a supplement to current CD8 T-cell vaccination regimes, as an approach to deposit Trm cells in tissues where local antigen presentation is a requirement. The two targeting antibodies we tested delivered antigen to either both respiratory DC subsets (anti-Clec12a) or the $\mathrm{CD}_{103^{+}}$subset alone (antiCD205). Both were capable of inducing Trm cell development that, in turn, afforded an elevated level of protection against a lethal influenza virus challenge. Intranasal delivery of targeting antibodies could both initiate T-cell priming and promote local presentation and Trm cell differentiation, although the population of Trm cells generated in this situation was smaller in comparison with the situation where preactivated effector cells were seeded into mice before mAb-OVA immunization. Furthermore, this population was not protective (data not shown). This would further support the use of ATV as a supplement to current $\mathrm{T}$ cell-priming regimes, or suggest multiple immunizations and boosting may be necessary to establish a sizable population of protective Trm cells.

In summary, we showed that the lung represents a tissue where local antigen recognition is required for Trm cell generation. Previous "prime-pull" vaccination strategies for Trm cell generation will not be effective at depositing Trm cells within this organ. For this and other tissues where there is an antigen dependency for Trm cell differentiation, we propose an extension of this approach-"prime, pull and present." This new approach, like its predecessor, relies on the induction of effector $\mathrm{T}$ cells and the topical application of inflammatory agents to pull the cells into the tissue, but also includes the local deposition of antigen within the tissue as a means to drive antigen-dependent Trm cell differentiation.

\section{METHODS}

Mice. C57BL/6, B6.SJL-Ptprc ${ }^{\mathrm{a}} \mathrm{Pep} 3^{\mathrm{b}} /$ BoyJ (CD45.1), H2-K ${ }^{\mathrm{bm} 1}$, and OT-I.CD45.1/2 mice were bred and housed in specific pathogen-free conditions in the animal facility at the Bio21 Molecular Science and Biotechnology Institute, the University of Melbourne. TGF $\beta$ Rflox/ flox $\times$ dlck $\times$ OT-I.CD45.1/2 mice were generously provided by $\mathrm{N}$. Zhang and M. Bevan and have been described previously. ${ }^{28}$ Bone marrow chimeras were generated by irradiation of recipient mice with two doses of 550 rads, $3 \mathrm{~h}$ apart, followed by reconstitution with $5 \times 10^{6}$ donor bone marrow cells. All experiments were done in accordance with the Institutional Animal Care and Use Committee guidelines of the University of Melbourne.

In vitro activation of OT-I cells. Purified OT-I CD8 ${ }^{+}$T cells were stimulated with peptide-pulsed splenocytes for 4 days in the presence of $20 \mathrm{U} \mathrm{ml}^{-1}$ interleukin-2. Mice were seeded with $5 \times 10^{6}$ in vitroactivated cells.

Infections and immunizations. Mice were infected intranasally with $10^{4}$ plaque-forming units of x31-OVA, Pr8-OVA (encode the $\mathrm{OVA}_{257-264}$ epitope within the neuraminidase stalk) or the parental strains $\mathrm{x} 31$ or $\operatorname{Pr} 8$ (viruses generously provided by S Turner, University 
of Melbourne, Melbourne, Australia). Mice were weighed daily and assessed for visual signs of clinical disease including inactivity, ruffled fur, labored respiration, and huddling behavior. Animals that lost $>20 \%$ of their original body weight and/or displayed evidence of pneumonia were killed. Titers of infectious virus in tissue homogenates were determined by standard plaque assay on MDCK cells in the presence of trypsin. Mice were given $1 \mu \mathrm{g}$ CCL 4 and CCL3, or $1 \mu \mathrm{g}$ of LPS or polyinosinic:polycytidylic acid, in a volume of $30 \mu \mathrm{l}$ of phosphate-buffered saline intranasally.

Intravascular staining. To label cells in the circulation, mice were given injection of $5 \mu \mathrm{g}$ phycoerythrin-conjugated anti-CD8 (53-6.7; BD Pharmingen, San Diego, CA) 5 min before killing as previously described. ${ }^{22}$ Mice were perfused with phosphate-buffered saline and tissues were harvested, processed, and stained with anti-CD8-APC (YTS-169), CD103 (2e7), CD45.1(A20.1), and CD45.2 (104).

Antibody administration. The anti-Clec12A and anti-DEC205 antibodies conjugated to OVA were engineered as previously described. ${ }^{25,33}$ Mice were administered $5 \mu \mathrm{g}$ anti-Clec12A (1/06-5D3) or anti-DEC205 (NLDC145) or isotype control (GL117) intranasally with $1 \mu \mathrm{g}$ of LPS 1 day after injection of $5 \times 10^{6}$ in vitro-activated OTI.CD45.1 cells. The recombinant antibodies will carry comparable amounts of OVA. Each antibody has been engineered so that one molecule of OVA ( $45 \mathrm{KDa})$ is genetically fused to the end of each heavy chain, hence each antibody ( 2 heavy +2 light chains; $150 \mathrm{KDa})$ will contain 2 OVA molecules $(90 \mathrm{KDa})$. Therefore, $5 \mu \mathrm{g}$ of targeting antibody instilled intranasally will contain $1.8 \mu \mathrm{g}$ of OVA protein.

Flow cytometry and cell sorting. Single-cell suspensions were prepared from spleens by mechanical disruption. Mice were perfused before harvest of the lung tissue that was enzymatically digested for $1 \mathrm{~h}$ at $37^{\circ} \mathrm{C}$ in $3 \mathrm{ml}$ of collagenase type $3\left(3 \mathrm{mg} \mathrm{ml}^{-1}\right.$ in RPMI-1640 supplemented with $2 \%$ fetal calf serum). Cells were stained for $25 \mathrm{~min}$ on ice with the appropriate mixture of mAbs and washed with phosphate-buffered saline with $1 \%$ bovine serum albumin. The conjugated $\mathrm{mAbs}$ were obtained from $\mathrm{BD}$ Pharmingen or eBioscience (San Diego, CA). Cells were sorted on an Aria III (BD Biosciences, San Diego, CA) to $>95 \%$ purity.

CFSE proliferation assay. A total of 25,000 CFSE-labeled naive OT-I cells were cultured in the presence of either 25,000 $\mathrm{CD}_{103}{ }^{+}$or $\mathrm{CD}_{11 \mathrm{~b}}{ }^{+} \mathrm{DCs}$, or macrophages or parenchyma cells sorted out of the lung $18 \mathrm{~h}$ after the intranasal delivery of $300 \mu \mathrm{g}$ of OVA protein and $1 \mu \mathrm{g}$ LPS in the presence or absence of $100 \mu \mathrm{g} \mathrm{ml}^{-1}$ neutralizing anti-TGF $\beta$ antibody (1D11; Walter \& Eliza Hall Institute Monoclonal Antibody Facility, Melbourne, Victoria, Australia). Proliferation was measured $60 \mathrm{~h}$ later.

Enzyme-linked immunosorbant assay. A total of 20,000 DCs were sort purified from the lungs of mice and cultured overnight with LPS. TGF $\beta$ enzyme-linked immunosorbant assay (eBioscience) for the detection of both biological and latent TGF $\beta-1$ was performed according to the manufacturer's instructions. The amount of TGF $\beta-1$ $\left(\mathrm{pg} \mathrm{ml}^{-1}\right)$ depicted is the amount detected minus the amount in the culture media.

Real-time PCR. For the measurement of Itgb8 expression, RNA was extracted with an RNeasy kit (Qiagen, Valencia, CA) from OT-I T cells (naive or day 3 after activation) of $\mathrm{CD}_{11 \mathrm{~b}}{ }^{+}$and $\mathrm{CD} 103^{+}$DCs sorted from the lungs of naive mice (as described above). Reverse transcription-PCR was done with a Sensifast cDNA synthesis kit according to the manufacturer's instructions (Bioline, Taunton, MA). Real-time PCR was performed with either $\mathrm{RT}^{2}$ qPCR Primer sets specific for mouse Itgb8 (Qiagen) or primers specific for GAPDH (5'-CCAGGTTGTCTCCTGCGACTT- $3^{\prime}$ and $5^{\prime}$-CCTGTTGCTGTA GCCGTATTCA-3') and Sensifast SYBR Lo-ROX Master Mix according to the supplier's recommendations (Bioline). Expression of Igtb8 was normalized to the housekeeping gene Gapdh.
Statistics. All data were analyzed using Prism 6 (Graphpad Prism, La Jolla, CA). Groups were analyzed by one-way analysis of variance, with Bonferroni's multiple comparisons post test comparing all groups with the control or an unpaired Student's $t$-test or log-rank (Mantel-Cox) test.

SUPPLEMENTARY MATERIAL is linked to the online version of the paper at http://www.nature.com/mi

\section{ACKNOWLEDGMENTS}

We thank M. Bevan and N. Zhang for TGF $\beta$ RII KO OT-I mice. This work was funded by the National Health and Medical Research Council (NHMRC) of Australia, with additional support from the University of Melbourne (Early Career Establishment Grant) and the Ramaciotti Foundation (to L.M.W.). J.A. Villadangos is a NHMRC Principal Research Fellow and L.M. Wakim is a NHMRC Career Development Fellow.

\section{DISCLOSURE}

The authors declared no conflict of interest.

(c) 2015 Society for Mucosal Immunology

\section{REFERENCES}

1. Brown, L.E. \& Kelso, A. Prospects for an influenza vaccine that induces cross-protective cytotoxic T lymphocytes. Immunol. Cell Biol. 87, 300-308 (2009).

2. Braciale, T.J. Immunologic recognition of influenza virus-infected cells. II. Expression of influenza A matrix protein on the infected cell surface and its role in recognition by cross-reactive cytotoxic T cells. J. Exp. Med. 146, 673-689 (1977).

3. Kees, U. \& Krammer, P.H. Most influenza A virus-specific memory cytotoxic T lymphocytes react with antigenic epitopes associated with internal virus determinants. J. Exp. Med. 159, 365-377 (1984).

4. Yewdell, J.W., Bennink, J.R., Smith, G.L. \& Moss, B. Influenza A virus nucleoprotein is a major target antigen for cross-reactive anti-influenza A virus cytotoxic T lymphocytes. Proc. Natl. Acad. Sci. USA 82, 1785-1789 (1985).

5. Veiga-Fernandes, H., Walter, U., Bourgeois, C., McLean, A. \& Rocha, B. Response of naive and memory CD8 + Tcells to antigen stimulation in vivo. Nat. Immunol. 1, 47-53 (2000).

6. Gebhardt, T. \& Mackay, L.K. Local immunity by tissue-resident CD8(+) memory T cells. Front. Immunol. 3, 340 (2012).

7. Purwar, R., Campbell, J., Murphy, G., Richards, W.G., Clark, R.A. \& Kupper, T.S. Resident memory T cells (T(RM)) are abundant in human lung: diversity, function, and antigen specificity. PLoS One 6, e16245 (2011).

8. Turner, DL et al. Lung niches for the generation and maintenance of tissueresident memory T cells. Mucosal Immunol. 7, 501-510 (2014).

9. Wakim, L.M., Gupta, N., Mintern, J.D. \& Villadangos, J.A. Enhanced survival of lung tissue-resident memory CD8 $(+)$ Tcells during infection with influenza virus due to selective expression of IFITM3. Nat. Immunol. 14, 238-245 (2013).

10. Wakim, L.M. et al. The molecular signature of tissue resident memory CD8 T cells isolated from the brain. J. Immunol. 189, 3462-3471 (2012).

11. Bevan, M.J. Memory T cells as an occupying force. Eur. J. Immunol. 41, 1192-1195 (2011).

12. Gebhardt, T., Wakim, L.M., Eidsmo, L., Reading, P.C., Heath, W.R. \& Carbone, F.R. Memory T cells in nonlymphoid tissue that provide enhanced local immunity during infection with herpes simplex virus. Nat. Immunol. 10, 524-530 (2009).

13. Mackay, L.K. et al. Long-lived epithelial immunity by tissue-resident memory $\mathrm{T}$ (TRM) cells in the absence of persisting local antigen presentation. Proc. Natl. Acad. Sci. USA 109, 7037-7042 (2012).

14. Schenkel, J.M., Fraser, K.A., Vezys, V. \& Masopust, D. Sensing and alarm function of resident memory CD8 $(+)$ T cells. Nat. Immunol. 14, 509-513 (2013).

15. Wu, T. et al. Lung-resident memory CD8 Tcells (TRM) are indispensable for optimal cross-protection against pulmonary virus infection. J. Leukoc. Biol. 95, 215-224 (2013). 
16. Liang, S., Mozdzanowska, K., Palladino, G. \& Gerhard, W. Heterosubtypic immunity to influenza type A virus in mice. Effector mechanisms and their longevity. J. Immunol. 152, 1653-1661 (1994).

17. Piet, B. et al. CD8(+) T cells with an intraepithelial phenotype upregulate cytotoxic function upon influenza infection in human lung. J. Clin. Invest. 121, 2254-2263 (2011).

18. Casey, K.A. et al. Antigen-independent differentiation and maintenance of effector-like residet memory Tcells in tissues. J. Immunol. 188, 4866-4875 (2012).

19. Wakim, L.M., Woodward-Davis, A. \& Bevan, M.J. Memory T cells persisting within the brain after local infection show functional adaptations to their tissue of residence. Proc. Natl. Acad. Sci. USA 107, 17872-17879 (2010).

20. Kohlmeier, J.E. et al. The chemokine receptor CCR5 plays a key role in the early memory CD8 $+\mathrm{T}$ cell response to respiratory virus infections. Immunity 29, 101-113 (2008).

21. Shin, H. \& Iwasaki, A. A vaccine strategy that protects against genital herpes by establishing local memory $\mathrm{T}$ cells. Nature 491 , 463-467 (2012).

22. Anderson, K.G. et al. Cutting edge: intravascular staining redefines lung CD8 T cell responses. J. Immunol. 189, 2702-2706 (2012).

23. Boscardin, S.B. et al. Antigen targeting to dendritic cells elicits long-lived $T$ cell help for antibody responses. J. Exp. Med. 203, 599-606 (2006).

24. Shortman, K., Lahoud, M.H. \& Caminschi, I. Improving vaccines by targeting antigens to dendritic cells. Exp. Mol. Med. 41, 61-66 (2009).
25. Lahoud, M.H. et al. The C-type lectin Clec12A present on mouse and human dendritic cells can serve as a target for antigen delivery and enhancement of antibody responses. J. Immunol. 182, 7587-7594 (2009).

26. Maamary, J. et al. Newcastle disease virus expressing a dendritic celltargeted HIV gag protein induces a potent gag-specific immune response in mice. J. Virol. 85, 2235-2246 (2011).

27. Lee, Y.T. et al. Environmental and antigen receptor-derived signals support sustained surveillance of the lungs by pathogen-specific cytotoxic $T$ lymphocytes. J. Virol. 85, 4085-4094 (2011).

28. Zhang, N. \& Bevan, M.J. Transforming growth factor-beta signaling controls the formation and maintenance of gut-resident memory $t$ cells by regulating migration and retention. Immunity 39, 687-696 (2013).

29. Mackay, L.K. et al. The developmental pathway for CD103CD8 tissueresident memory T cells of skin. Nat. Immunol. 14, 1294-1301 (2013).

30. Hu, Y. \& Cauley, L. Antigen and transforming growth factor Beta receptors contribute to long term functional and phenotypic heterogeneity of memory CD8 T cells. Front. Immunol. 4, 227 (2013).

31. Yu, C.I. et al. Human CD1C + dendritic cells drive the differentiation of CD103 + CD8 + mucosal effector T cells via the cytokine TGF-beta. Immunity 38, 818-830 (2013).

32. Lahoud, M.H. et al. Targeting antigen to mouse dendritic cells via Clec9A induces potent CD4 T cell responses biased toward a follicular helper phenotype. J. Immunol. 187, 842-850 (2011).

33. Bourges, D. et al. Targeting the gut vascular endothelium induces gut effector CD8 T cell responses via cross-presentation by dendritic cells. J. Immunol. 179, 5678-5685 (2007). 